This item was submitted to Loughborough's Research Repository by the author.

Items in Figshare are protected by copyright, with all rights reserved, unless otherwise indicated.

\title{
Use of thin laminar liquid flows above ablation area for control of ejected material during excimer machining
}

PLEASE CITE THE PUBLISHED VERSION

PUBLISHER

Professional Engineering Publishing / @ IMechE

VERSION

VoR (Version of Record)

LICENCE

CC BY-NC-ND 4.0

\section{REPOSITORY RECORD}

Dowding, Colin F., and Jonathan Lawrence. 2019. "Use of Thin Laminar Liquid Flows Above Ablation Area for Control of Ejected Material During Excimer Machining". figshare. https://hdl.handle.net/2134/6399. 
This item was submitted to Loughborough's Institutional Repository (https://dspace.lboro.ac.uk/) by the author and is made available under the following Creative Commons Licence conditions.

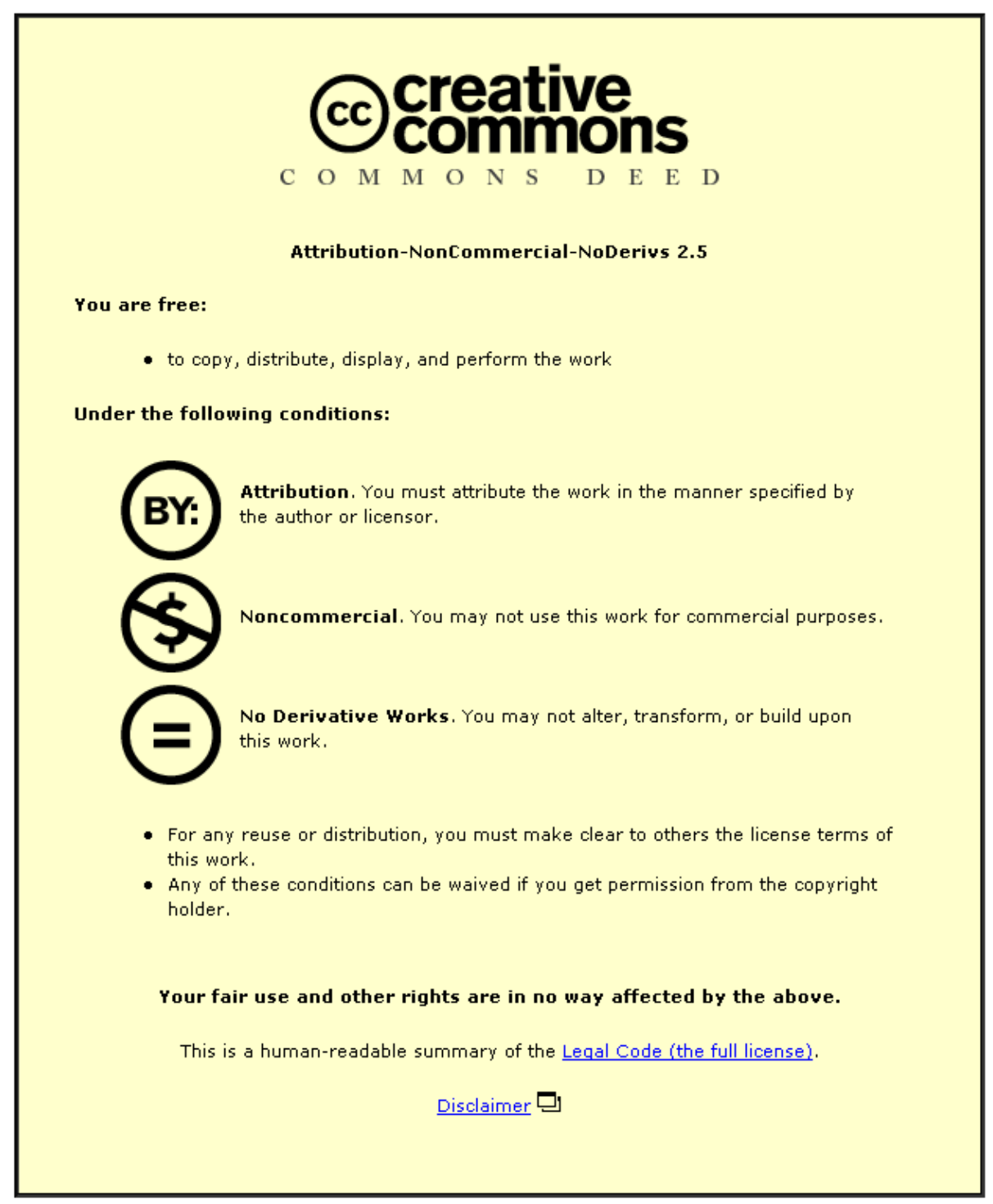

For the full text of this licence, please go to: http://creativecommons.org/licenses/by-nc-nd/2.5/ 


\title{
Use of thin laminar liquid flows above ablation area for control of ejected material during excimer machining
}

C F Dowding* and J Lawrence

Wolfson School of Mechanical and Manufacturing Engineering, Loughborough University, Loughborough, UK

The manuscript was received on 12 January 2009 and was accepted after revision for publication on 13 February 2009.

DOI: 10.1243/09544054JEM1479

\begin{abstract}
To observe excimer laser machining through thin liquid films and the effects thereof on debris control, equipment was designed to contain a small control volume that can be supplied with a laminar thin film of de-ionized (DI) water to flow over the workpiece. Using the same equipment, comparison with non-liquid ablation was possible. Reliable calculations of the debris size and density with respect to the distance from the centre of the shot, as well as the identification of modal trends in the dispersion of the debris were obtained from analysis of microscope images with graphical analysis software. The results suggest that the positional debris deposition of samples machined in ambient air show a modal tendency that is reliant on the machined feature shape and is a function of species size. This is proposed to be due to the interaction of multiple shockwaves at the extent of ablation plumes generated at geometryspecific locations in the feature. Debris is deposited where the shockwaves collide. Large debris did not typically travel much further than the boundary of the machined feature, whereas intermediate debris was found in radial streaks at a normal to the circular feature's perimeter. Laminar flows of liquid have shown potential to modify the end position and typical size of the debris produced, as well as increased homogeneity of deposition density. The use of immersion has reduced typical range by 17 per cent, and the deposition within the boundary of the ablation plume has a comparatively even population density with respect to the sample machined in ambient air. Outside the ablation plume extents, evidence of positional control of deposited debris species by laminar flow DI water immersion is shown by debris deposition in rippled flow line patterns for both circular and square features, indicating the action of transport by fluid flow. A typical increase in debris size by an order of magnitude when using DI water as an immersing liquid has been measured, a result that is in line with a colloidal interaction response.
\end{abstract}

Keywords: excimer, ablation, debris control, liquid, polymer

\section{INTRODUCTION}

Micro particulate ablation debris produced during excimer laser beam interaction with a material are highly excited objects of very small mass. This means that these particles need a large force to decelerate them, yet they only have a very small surface area available for a decelerating force to be applied. Traditionally excimer laser machining has been conducted in gaseous mediums: these are easy and cheap to

*Corresponding author: Wolfson School of Mechanical and Manufacturing Engineering, Loughborough University, Loughborough, Leicestershire LE11 3TU, UK.

email: c.f.dowding@lboro.ac.uk contain, regulate, and supply. These mediums, however, have very low viscosities and consequently cannot provide high drag force to the small, highly excited particles moving through them. Conversely, liquids have a much higher viscosity than gases; thus the small particles will face much more resistance. Additionally, liquids are better conductors, dissipating electrostatic forces whilst completely removing capillary adhesion problems by swamping condensation sites, which in turn promotes surface cleaning. Despite these benefits to debris control there are potential problems posed by liquids to the beam path: reflectance, uneven refraction, micro-bubbles, transmissivity, and heat-related effects are all possible. 
Debris is generated as either a solid or as a melt which later solidifies either during transit or after coming to rest. Both of these are generated by different mechanisms that work together simultaneously [1-9]. However, it is generally accepted that the motive force for these species, once separated from the substrate, is the ablation-generated vapour plume and its associated shockwave $[\mathbf{1 0}, \mathbf{1 1}]$.

As already stated, a number of ablation mechanisms appear to be at work at any one time: photochemical interactions produce gaseous plumes [9] and melt, presumably generated by a non-photochemical interaction, such as sub-surface superheating [10], this is accelerated by the shockwave that expands away from the ablation spot until gaseous matter is no longer generated at a sufficient rate by the photochemical beam interaction with the substrate. At this point the plume begins to recede, the shockwave collapsing with it, allowing the melt to continue on its own trajectory. The non-propelled trajectory is governed by longrange adhesion forces such as van der Waals and coulomb electrostatic forces dependent on the proximity of the debris to local macroscopic items [12], additional to this is the minor effect of gravity, dependent on the orientation of the sample and lastly the drag generated by the movement of the debris through its environment.

Solids are most likely to be produced by acoustically generated stresses and the shear forces these generate $[\mathbf{9}, \mathbf{1 3}]$, causing rupture and dissociation of material in the form of small chunks with the same chemistry as the original substrate. Once separate from the substrate, the mode of acceleration for these species is identical to that of the melt. This means that there are five critical parameter groups involved in the time of flight of any debris:

(a) beam characteristics, such as wavelength [14], shape [9], power [15], shot duration $[\mathbf{9}, \mathbf{1 6}, \mathbf{1 7}]$, and temporal shape [13];

(b) material chemistry $[\mathbf{9}, \mathbf{1 5}]$;

(c) environmental characteristics $[\mathbf{1 2}, \mathbf{1 8}]$, such as chemistry, pressure, density, and viscosity;

(d) adhesion forces comprised of environment conductivity $[\mathbf{1 2}, \mathbf{1 9}]$ and van der Waals attraction [12];

(e) surrounding geometry such as proximity of surrounding macro objects and the orientation of ablation spot compared to the gravitational vector.

Debris control during excimer laser micromachining is a critical subject for future progression. To this end, this paper focuses exclusively on the production of debris, regardless of the effect of liquid immersion on the machining quality and ablation rate of features produced. Obviously, the laser fluence could influence the debris size and spatial distribution; but, this work concentrates solely on determining whether a thin laminar flow of de-ionized water across bisphenol A polycarbonate affects the debris size and spatial distribution of particles generated by excimer laser ablation. The function of thin laminar flows in this respect will be compared to the debris characteristics of a sample machined in air using identical laser parameters.

Bisphenol A polycarbonate, commonly referred to by its trade name: Lexan, amongst other polymers with similar properties is now being adopted as a durable and lightweight substitute for glass with particular relevance for the portable display sector [20]; thus it will be the focus of this work. The most common current methods for removal of debris are post-process cleaning of the sample either by laser cleaning processes, chemical processes, or ultrasonic bathing $[12,21,22]$. These are extra steps in the manufacturing process and could present possibility for damage of components that have already had the investment of expensive micromachining techniques made upon them. Typically the use of vacuum or positive pressure gas jetting during manufacture have been found lacking, either presenting further engineering challenges to the process equipment such as vibration; or to be ineffectual, merely moving the debris to another location on the substrate [23].

Laminar flow liquid immersion uses the principle of increased density and viscosity over traditional gaseous machining environments to entrap all debris particles of both melt and solid state as they are launched by the ablation plume and retain them by the action of surface tension, preventing deposition of the debris on the substrate. This technique is intended to improve on gas-assisted debris control techniques by removing the debris from the beam path between shots in the same way, but then retain the debris without deposition. This 'in-line' method will potentially offer an exceptionally clear beam path for every laser shot, as well as removing the need for costly post-process cleaning techniques.

\section{BACKGROUND THEORY}

The use of a liquid immersion technique to control ablation-generated debris is borne from an understanding of the effect that liquid mediums have on adhesion forces along with techniques used to capture laser-ablation-generated nanoparticles [24]. Liquid immersion affects all of the primary adhesion forces: van der Waals, capillary, and electrostatic. It also has an effect on the ablation mechanism and the propagation of the subsequent ablation plume.

\subsection{Adhesion theory}

The force most commonly identified as an adhesion force bears its discoverer's name - van der Waals 
(FvdW) also known as the 'secondary valence force'. This is the primary force of adhesion acting upon small (less than $50 \mu \mathrm{m}$ diameter) particles lying upon a dry surface [12]. This can be increased in strength due to deformation of the particle, the surface, or both, since all act to increase the surface area in contact between the two [12]. Particles less than $1 \mu \mathrm{m}$ diameter can be held to surfaces by forces exceeding 100 dynes or roughly $109 \mathrm{dyn} / \mathrm{cm}^{2}$.

The tendency for two bodies to adhere to each other purely through the action of FvdW can be described by using either the Hamaker or Lifshitz constant [21]. The Hamaker option is an approximation [25], whereas the Lifshitz technique is more accurate, as it takes into account the effect one charge can have on a neighbour. The tendency for two bodies to be attracted by these forces varies greatly with material. The general trend being that metals have high values and polymers possess much lower coefficients. The system involved also has an effect, where homogeneous pairings generally experience greater adhesion force. Critically it has been found that immersion in fluids, such as water, can drastically reduce the attraction between a homogeneous paring for most materials [26]. For the purpose of laser micromachining most adhesion interactions will be between a homogeneous pair; thus the use of liquid and in particular water immersion becomes attractive.

In a dry system, electrostatic forces act to hold particles to surfaces in partnership with FvdW; they can also play a prominent role in attracting the particles to the surface before adhesion. There are two main electrostatic forces that act between particles and surfaces: electrostatic image forces and electrostatic double layer forces. The long-range effect of electrostatic image forces act to attract a drifting particle towards another body. This effect is mainly dependent upon the charge stored on the two bodies, the distance between their charge centres, and the dielectric constant of the medium in which they reside [12]. It has been shown both theoretically and experimentally that the difference in potential difference between the two bodies is critical in the attraction between them [12].

Once two bodies are in contact the electrostatic double layer force takes control. This can be attractive or repulsive dependent on the characteristics of the medium surrounding the bodies. This interaction depends greatly upon the contact potential difference. When separated by a medium of the correct dielectric nature a Yukawa repulsion [19] can be instigated, where the medium conducts a small proportion of the charge of one comparatively large body (such as the substrate) to the small adhering body (the particle) thus causing both to have a charge of the same sign and causing electrostatic repulsion.
A medium of high conductivity is preferable for this effect and again suggests that the use of a liquid medium such as water has potential benefits for lowering total adhesion force.

Capillary condensation is a highly environmentsensitive adhesion phenomenon. In humid conditions on a smooth surface it can exceed the force delivered by both FvdW and electrostatic interactions. Capillary adhesion can only occur when the particlesurface system resides in a primarily gaseous medium but can remain active even after significant measures have been taken to evaporate the meniscus generating the adhesion [27]. The meniscus responsible for the adhesion is generated via the action of capillary condensation of a liquid vapour present in a primarily gaseous medium around a seed point, often a surface asperity, the surface tension of the resultant meniscus is what provides the attractive force; thus a totally dry environment removes the possibility of generating a new meniscus and a smooth surface offers smaller seed points for a meniscus to grow. Interestingly, a critical humidity value must be achieved to generate significant meniscus adhesion: approximately 60 per cent $\mathrm{RH}$ for most mediums [28]. Once capillary adhesion takes hold it tends to increase the total adhesion force by a factor of five to seven [29]. The addition of a liquid to a particle substrate system can have the result of causing repulsion between the two bodies. This force is generated by the electrostatic double layer interaction covered above. However, in this case the medium itself is the main factor and the interaction can be predicted using a model developed by Vezenov et al. [30]. The addition of liquid immersion also lowers the viscosity difference between the meniscus and the medium when compared to a meniscus/air system, thus whilst immersed the action of capillary force is greatly reduced as the surface tension generated between the particle and surface by the meniscus is minimized [12].

As has been evidenced above, there is a strong argument for the use of liquid immersion by a conducting fluid as an effective method for reducing FvdW forces and negating both electrostatic image and capillary forces until machining has ended and the workpiece is removed from the immersion environment. However, immersion of the workpiece has more potential debris benefits, both in terms of debris chemistry and in the entrapment of the particles as they accelerate away from the ablation spot.

\subsection{Effect of immersion on ablation}

The mechanism of ablation in liquids has some significant differences from ablation in gases or a vacuum. The most important of these is that the liquid confines the expansion and movement of the 
plasma plume due to its higher density and viscosity. This confinement can greatly influence the thermodynamic and kinetic properties of the plasma and therefore the evolution of the plume. This can give rise to distinctly different scenarios both during vaporization and condensation when compared to ablation in gaseous or vacuum environments [31].

Fabbro et al. [32] found that a shockwave is induced by the laser-induced plasma and is confined to the extents of the plume by the surrounding liquid. The laser-induced plasma, generated in Fig. 1(a), adiabatically expands when the plasma absorbs the latter part of the laser pulse and receives a further supply of vaporized mass from the target material at a supersonic velocity to create a shockwave as described in Fig. 1(b). This shockwave will generate increased pressure within the laser-induced plasma plume. This increase in pressure generated by the shockwave has become known as the 'plasmainduced pressure'. Thermodynamically, the increase in pressure will be coupled to an increase in temperature of the plasma; thus a laser-induced plasma under the confinement of the surrounding liquid has a higher temperature, pressure, and density than that of a plasma generated in a gaseous or vacuum environment, giving rise to more complex chemical interactions within the plume as displayed in Fig. 1(c). Lastly, the laser-induced plasma cools and condenses, as depicted in Fig. 1(d). A proportion of the plume will condense and settle as a film back onto the target material during plasma quenching by the confining liquid; this would be encouraged by the pressure applied on the plume by the confining liquid. The rest of the plume will condense and become entrapped by the confining liquid, resulting in the synthesis of small particles that rise to the surface of the liquid film due to the action of surface tension.

Sattari et al. [33] used ablation of ceramics immersed in 4 to $6 \mathrm{~mm}$ of distilled water running at a constant flow rate of $116 \mathrm{ml} / \mathrm{min}$ to produce nanoparticles. According to Zhu et al. [34] and Dowding and Lawrence [35], this depth of immersion liquid fits a thick film immersion ablation regime. Sattari et al. found particle productivity to be proportional to liquid flow rate because debris is removed from the site of production faster, allowing new nanoparticles to be formed by a following laser pulse. Also, laser fluence was found to be inversely proportional to the size of nanoparticles produced, supporting the dominance of a photomechanical etching mechanism of a confined ablation plume. Again, using a thick film regime, Katto et al. [36] used an excimer laser beam with pulse fluence ranging from 7 to $23 \mathrm{~mJ} / \mathrm{cm}^{2}$ to ablate hydroxyapatite under a stagnant immersion fluid of purified water to show that the dominant size of particles produced is governed by the wavelength of the laser, a result common with traditional laser
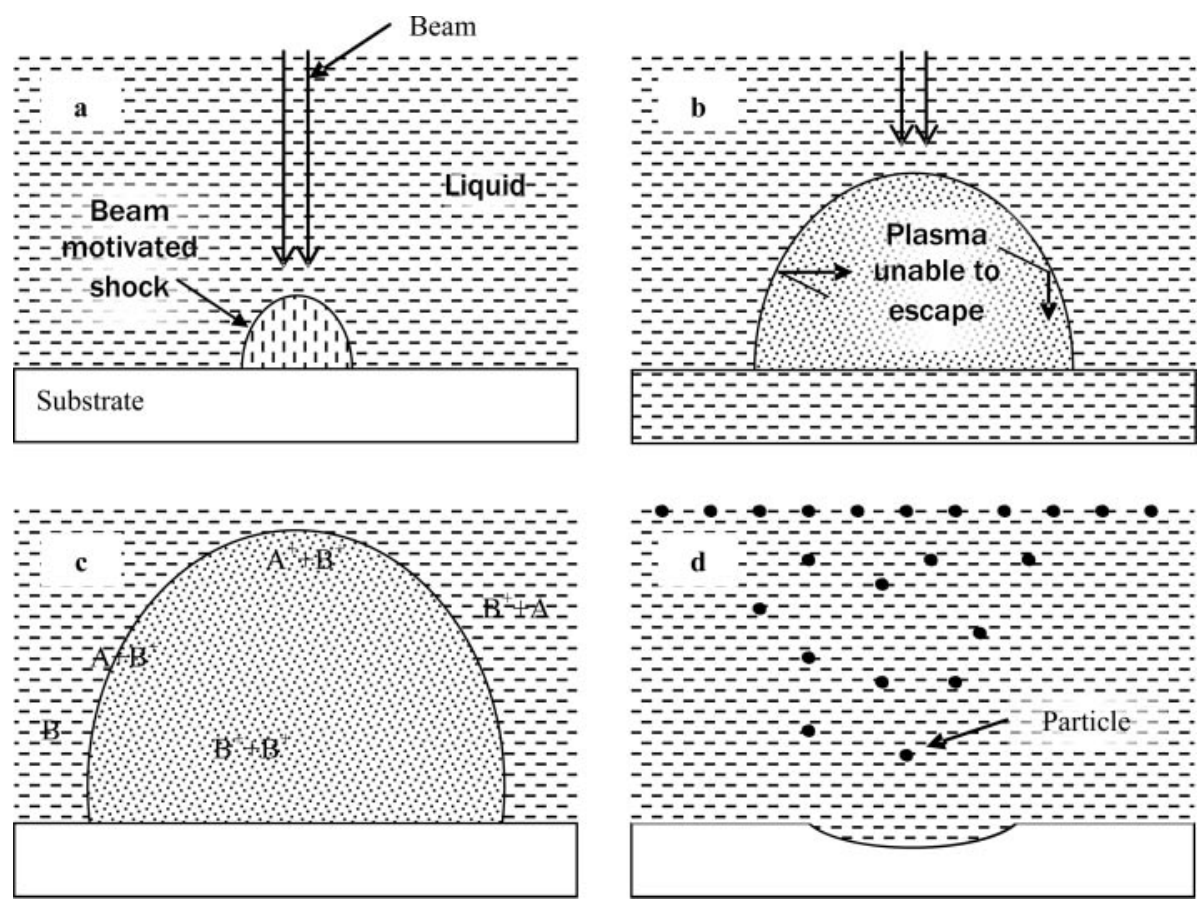

Fig. 1 Four stages of immersion ablation: (a) beam interaction, (b) confined expansion, (c) chemical reaction, and (d) colloidal particle formation [32] 
machining in gaseous mediums. The results of Katto et al. differ from those of Sattari et al., insofar as the Katto et al. results find that fluence is proportional to debris size: using a low fluence in the range specified above, ablation of the immersed target typically produced nanoparticles with a diameter less than $1 \mu \mathrm{m}$ and use of medium fluence ablation produced particles with a diameter greater than $1 \mu \mathrm{m}$. Katto et al. also reported sponge-like products when using the highest beam fluence in the range specified. This contrast in results is interesting in two ways: it suggests the use of a flowing immersion liquid is important in the beam-material interaction; and given that the ablation mechanism occurring is modified by the introduction of a liquid medium, can cause a change in debris characteristics that are significant to the function of thin laminar flow liquid debris control of ablation-generated debris because of the function of fluid drag by the flow on a debris particle being directly proportional to the size of the debris particle involved.

\subsection{Explosion mechanics}

The increased density and viscosity of a liquid medium can be used in the favour of debris control to decelerate particles flying away from the ablation spot after the collapse of the vapour plume. The displacement of every particle occurs in three separate phases with respect to time. The first, denoted $t_{\mathrm{v}}$ in Fig. 2, is the acceleration of the particle by expanding gas produced during ablation [13, 15-17, 37], the second phase, denoted $t_{\text {rec }}$ in Fig. 2, is the deceleration of the particle by recoil condensation of the gas after ablation has ended [15], immediately following the initial phase. Finally, the drag phase, denoted $t_{\mathrm{d}}$ in Fig. 2, comes into effect and the particle is assumed to be moving with respect to its surrounding medium and is

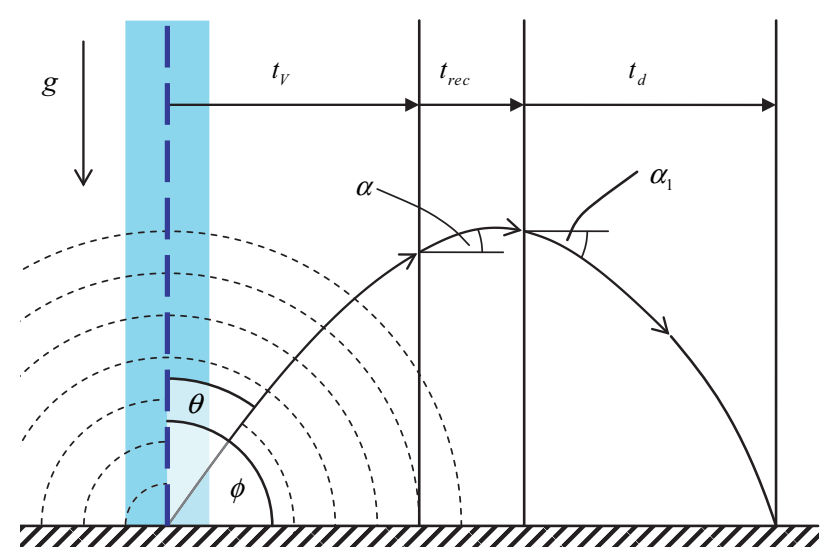

Fig. 2 Three critical stages of debris dispersion: plume acceleration, recoil deceleration, drag deceleration subjected to a fluid drag force by that medium. This model assumes all the particles are static before acceleration begins and the particles are accelerated at the front of an explosion pressure wave; that is, there is no drag force present during initial acceleration. Increasing the drag after the explosion and recoil phases have ended will reduce the distance travelled during the time frame denoted $t_{\mathrm{d}}$ in Fig. 2; stopping deposition on optics and allowing the work of surface tension to become useful and prevent the particles from being deposited on the substrate.

With the knowledge presented thus far it is clear that liquid immersion ablation offers reduced possibility of ablation-generated debris becoming deposited on the substrate by minimizing the long-range adhesion forces acting on the flying debris; for if debris does become deposited, the possibility of its removal is greatly increased by a reduction in contact adhesion forces. This proposal is bolstered by evidence showing that the chemistry of ablationgenerated debris can be controlled to produce a less reactive substance that will become entrapped in the immersing liquid. Equipment has been developed to explore this theory.

\section{EXPERIMENTAL PROCEDURES}

\subsection{Material}

Bisphenol A polycarbonate (Holbourne Plastics, Ltd) was as received in $1200 \mathrm{~mm} \times 1000 \mathrm{~mm}$ sheets of $0.5 \mathrm{~mm}$ thickness. Prior to excimer laser processing, the bisphenol A polycarbonate sheet was cut into rectangular sections of $30 \mathrm{~mm} \times 10 \mathrm{~mm}$ using scissors, as this is a shear cutting technique which avoids production of debris. Protective cover sheets were then peeled off each sample. Polycarbonate can be used either as a lens array medium or as a substrate for deposited thin films such as indium tin oxide [38].

\subsection{Laser processing}

An excimer laser (EMG 203 MSC; Lambda Physique, $\mathrm{GmbH}$ ) using $\mathrm{KrF}$ as the excitation medium was used to produce a beam with a wavelength of $248 \mathrm{~nm}$. Thereafter the beam was supplied to a laser micromachining optics assembly (EX-PS-500; Exitech, Ltd), where the raw beam was homogenized in the $x$ - and $y$-axis before being passed through either a stainless steel mask to produce a $7.5 \mathrm{~mm}$ square or a $7.5 \mathrm{~mm}$ diameter circular objective beam. The masked beam was then demagnified by $15 \times$ to produce a $500 \mu \mathrm{m}$ ablation spot size with a depth of focus (DoF) of $6 \mu \mathrm{m}$.

As shown in Fig. 3, the sample was mounted on a stainless steel table by means of the capillary action of isopropyl alcohol which was applied to the sample 


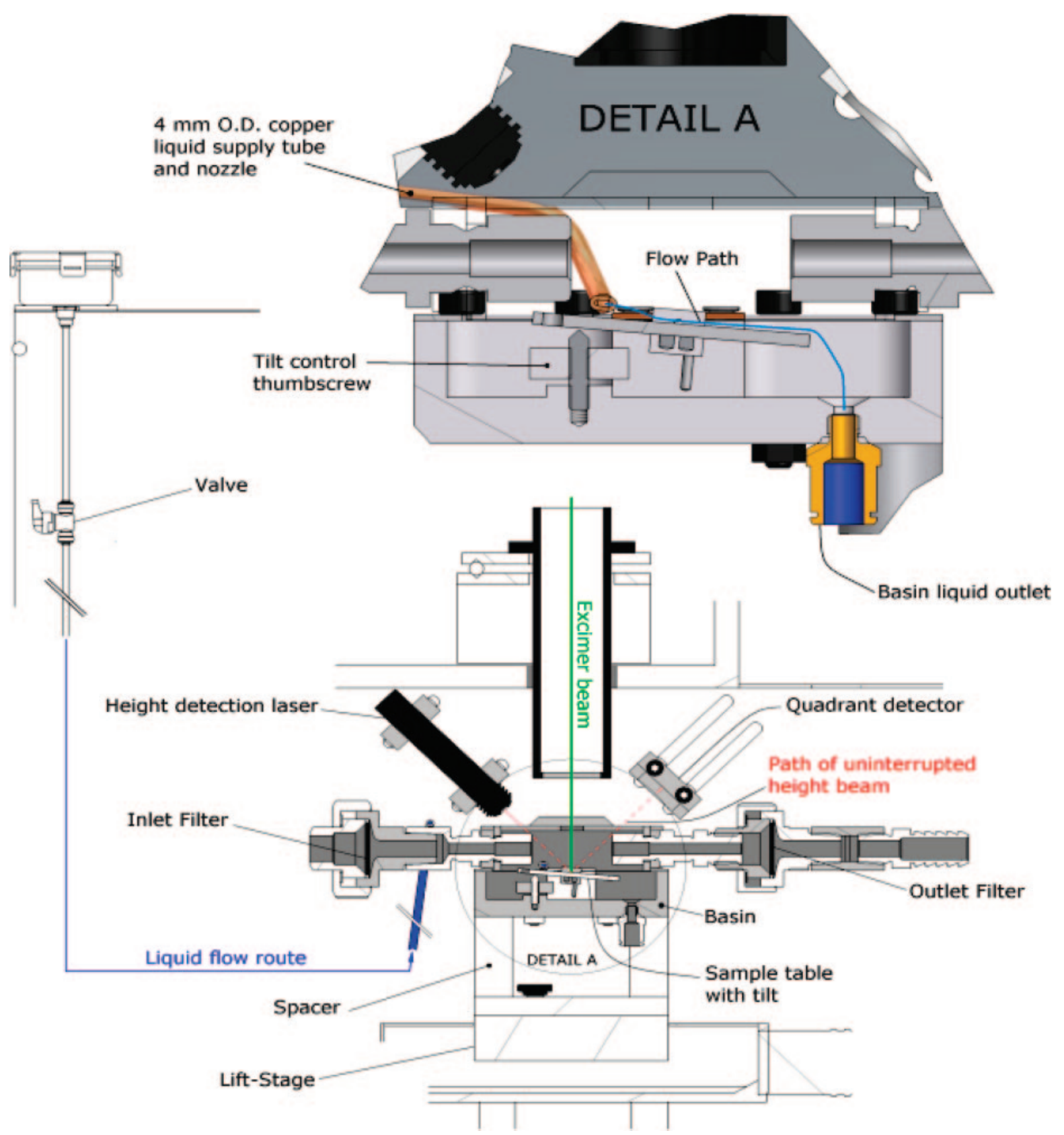

Fig. 3 Schematic of liquid immersion rig

table before the sample was mounted. The table was located to a pivot cradle by a tongue and groove arrangement twined with a dowel for linear constraint magnets allowed simple and secure repeatable fixing between the table and cradle. The cradle allowed the table to be tilted around its mid-point by use of an adjustable thumb screw that supported the water inlet end of the table.

This tilt mechanism had two experimental applications. First, it allowed the angle of incidence between the excimer laser beam and the sample to be modified. Second, it allowed the run-off rate of the liquid to be changed independently of the supplied liquid flow rate; hence the film thickness on the sample could be controlled. For this work, liquid was supplied by use of gravity feed from a butt supported at a constant height, thus a single flow rate was used for supply that was measured using a flow metre (F4500, Key Instruments) to be $0.015 \mathrm{~L} / \mathrm{s}$. For this work the sample tilt angle was left at $90^{\circ}$ to the incident beam, as this arrangement explores the most simple arrangement, easily reproducible for an industrial application. The supply was gated by a valve to allow rudimentary control of liquid flow before being delivered though a flat nozzle mounted $0.5 \mathrm{~mm}$ above the sample. The film thickness achieved with this arrangement was $0.8 \mathrm{~mm}$, measured 
using a graticule and magnifying optics. The sample table and water nozzle were positioned over a miniature basin with a hose leading to a sealed storage tub positioned a distance below the basin, again using gravity feed to ensure evacuation of liquid from the basin.

The basin geometry also provided location for a clear Perspex enclosure within which a controlled environment for experiments could be maintained. This enclosure provided a repeatable location for the water nozzle with respect to the basin and therefore sample table. The enclosure also had access and location for a gas inlet and outlet manifold. These manifolds allowed the installation of $25 \mathrm{~mm}$ diameter fibre mesh filters (AAWP02500; Millipore, Inc.) to remove airborne particles inside the enclosure or particles generated during ablation to be captured for subsequent inspection. This extraction system worked by the use of negative pressure; utilizing a vacuum pump mated to the outlet manifold.

During normal open use the rig was mounted upon a driven lift stage that was adapted to dynamically adjust the sample height dependent upon a signal generated by logical interpretation of the deflection of a reflected reference beam. However, with the inclusion of the Perspex enclosure for these experiments, the reference beam was obstructed and this dynamic focussing method could not be used. Instead the stability of the stage was trusted; that is, the sample height is set before the experiment to being as close to the centre of the DoF as possible. The feedback system was then overridden and the enclosure was installed before the experiment commenced. The DoF also played an important role in limiting the tilt angle (for a $500 \mu \mathrm{m}$ diameter spot size the maximum tilt angle possible whilst keeping the entire image inside focal tolerance is $1.36^{\circ}$, assuming the centre of the image lies at the pivot axis). Each sample was used for only one test to prevent crosscontamination. After lasing ended the enclosure was removed and the sample placed into a cell of a sealed sample tray to protect it from atmospheric dust

\subsection{Analysis techniques}

All samples described in this work are machined using an excimer laser beam with a profile described by Fig. 4. This profile was obtained using a beam profiler (SP620U; Spiricon, Ltd), imaging a fluorescing plate lying just above the mask plane. Machining was always conducted using a nominal $300 \mathrm{~mJ}$ beam at the shutter, which corresponds to approximately $6.5 \mathrm{~mJ}$ at the image once masked.

The large area of interest along with the high resolution necessary to register small debris conspire to required nine sector micrographs to be taken of each sample; sector micrographs were imaged under

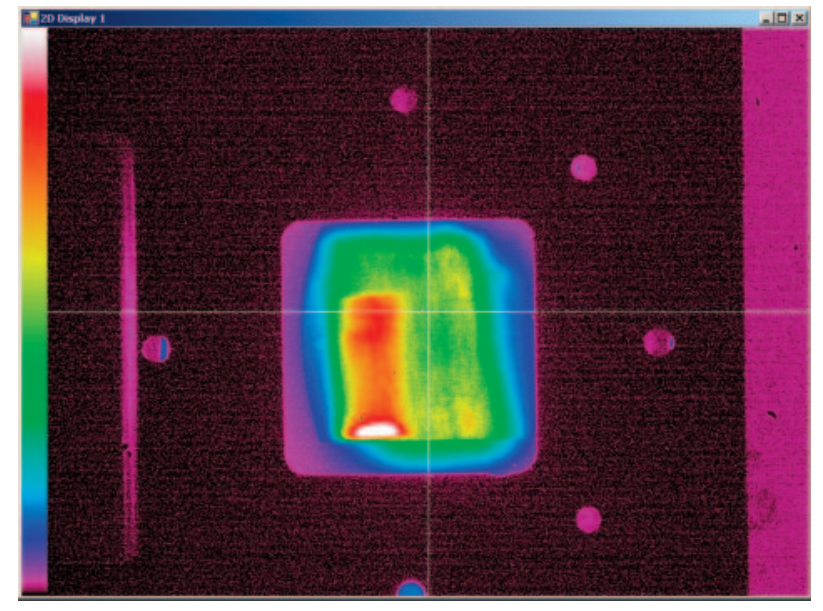

Fig. 4 Profile of beam used taken before samples produced

an optical microscope (MeF3; Reichert-Jung) fitted with a digital camera (Fujix HC300Z; Fujifilm). The 'Photomerge' tool of Adobe Photoshop was then used to stitch the sectors into large area, high resolution 24 bit colour micrographs. Micrographs were manipulated using graphical analysis software (Visilog Xpert 6.6; Noesis). Images were converted to an optimized greyscale with high local contrast using a 'lightness' algorithm. Variations in lighting across the image were then corrected using a 'lequalize' function. This data could now be binarized using the 'threshold' function. Careful binarization generated a digital map, identifying all debris images as 'high' signals and everything else as a 'low'. This data could be processed using the 'IAnalyse' tool to produce a table of data listing feature area, perimeter, and centre position. This was the data used in this publication to characterize the behaviour of debris.

\subsection{Data processing}

All plots of debris features identified by the binarization process were classified by area against $x-y$ position. All data was listed by pixel position that can be converted to an SI measure by a conversion factor. This has been taken using a graticule on the same microscope, so a pixel is equivalent to $0.46 \mu \mathrm{m}$. This conversion has been generated by dividing the number of pixels imaged over a distance of $1 \mathrm{~mm}$. The size of the debris has been grouped into shaded classes, the same legend, which is displayed in Fig. 6, is used for all results.

To reduce noise two result filters have been used. Any sample registering an area lower than 1 square pixel has been omitted. This action has been taken to lower the number of data points erroneously identified by lighting inconsistencies generated by the surface roughness of the sample material. Pixels recorded 
towards the edges of each section image taken by the microscope display this erroneous result proportionally with increasing distance from the section centre. This was because of the incident angle of the image illumination: as the incident angle increased, the presence of microscopic dimples and bumps on the material surface gave increased response. This could be partially rectified by stitching, this involved overlapping sections, thus averaging the light levels along the intersecting section seams; however, the outer edge of the stitched image could not be corrected in this fashion and the 'lequalize' algorithm was not capable of identifying and removing such features. This collection of circumstances meant significant noise at the outer edges of the stitched image. The second filter removed any item identified as greater than 1001 square pixels. These could be deemed an analysis anomaly where a number of separate debris items have been identified as a single object. This could occur when a number of particles lie within close proximity to each other and there was little contrast at the separate particle edges. Visilog classified these as a single item.

\section{RESULTS AND DISCUSSION}

Two feature geometries have been chosen as the deposition pattern of the debris appears to be geometry dependent. Both sets of features, machined in ambient air and under immersion, are machined with the sample table lying at a normal to the vector of the beam. This way all samples produced are not affected by the possible effects of incident beam angle. It must be noted that the mechanism of ablation is significantly modified by the implementation of a liquid medium rather than that of air, as is typical; this fact is alluded to above. The ablation threshold is modified, dependent upon film thickness, along with ablation rate. Dowding and Lawrence [35] detail the relationship between the ablation mechanism and the thin film fluid immersion generated by this experimental apparatus, finding that this equipment generates an increase in ablation threshold of approximately double and a decrease in ablation rate of 20 per cent. A circular feature has been chosen with the intention of producing debris deposition in an annular pattern to give a reasonable indication of mean range with respect to fluence. A square feature has been chosen to represent the modal trend of debris deposition for features with acute angle corners.

\subsection{Circular feature geometry}

The debris distribution of the sample machined in ambient air displays areas of localized high debris

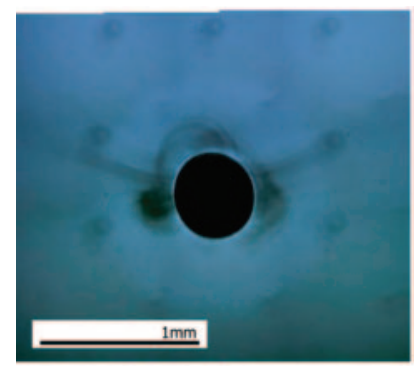

(a)

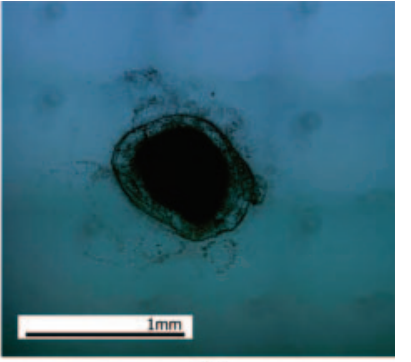

(b)
Fig. 5 Stitched section images of the ambient (a), and immersion (b) machined samples

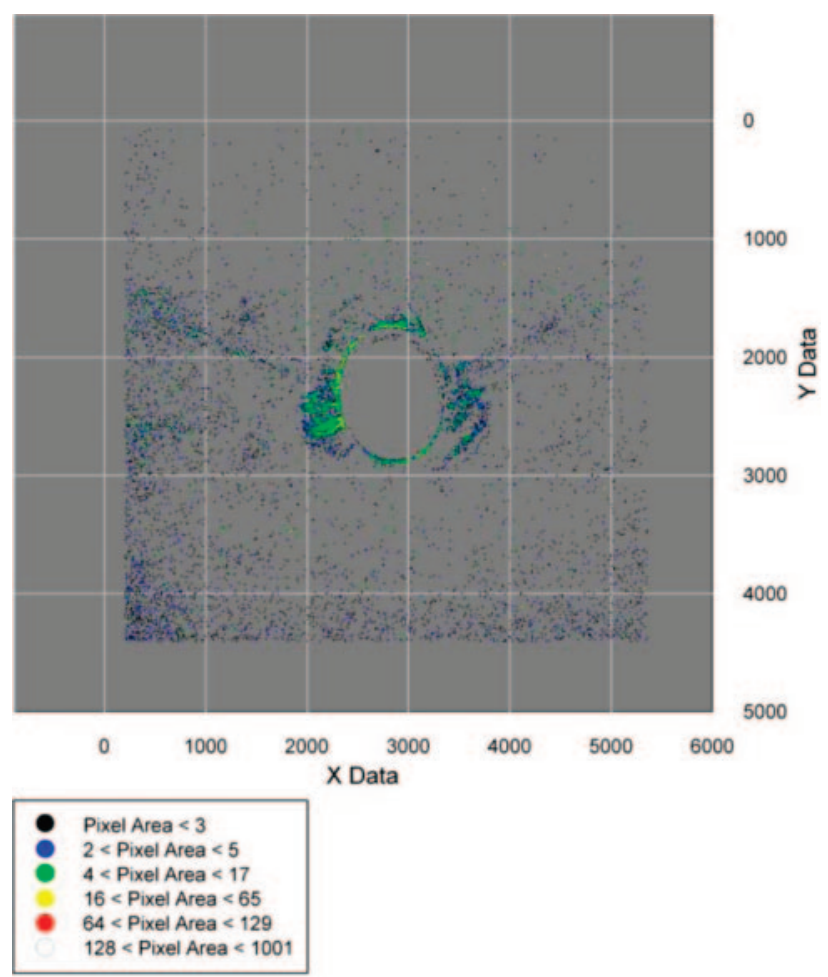

Fig. 6 Plot for circular feature showing debris size against position for a sample machined in ambient air

density as can be seen in Figs 5 and 6. Given the five variables available from the body of literature, one can say that there are three possible explanations for this occurrence; either working exclusively or in combinations thereof. The beam profile may have caused an uneven ablation rate across the image, creating an uneven pressure profile in the ablation plume; thus debris is consistently expelled with positional tendencies. Also, the surface profile of the original material may have generated localized changes in the beam incident angle; thus modifying the local trajectory of the debris. Finally, the material composition of the polycarbonate will not be completely homogeneous across the whole image; thus some areas within the image may produce more 
debris than others. Inspection of the original micrograph displayed in Fig.5(a) in concert with the Visilog analysis in Fig. 6 would tend to support the postulation that the deposition of debris is dominated by the shape of the ablation plume: two curved 'horns' can be seen extending with a large radius, symmetrically from the centre of the image.

The majority of the debris deposited lies within $180 \mu \mathrm{m}$ of the centre of the image. The largest of the debris produced (pixel area between 16 and 65) has very little range, lining the edge of the feature, large intermediate debris (pixel area between four and 17) appears to have less range than smaller species and lies in radial streaks from the edge of the feature. Both of these results are contrary to expectation; however, large intermediate debris also occurs in increased concentration along the horns, suggesting that debris is transported on the edge of the ablation plume, providing support for the theories of Dyer and co-workers [13, 15-17, 37].

When compared to the sample machined in ambient air, the sample produced under liquid immersion shows a more homogeneous distribution of debris. It must be noted that the dark line of debris surrounding the machined feature clearly visible in Fig. 5(b) have been filtered out of the data plotted in Fig. 7 - these separate debris items were generating signals with areas greater than 1001 square pixels, as the low contrast between individual items made groups appear to be single objects after the binarization process. Nevertheless, the raw image displayed in Fig. 5(b) is still useful as it clearly shows a boundary

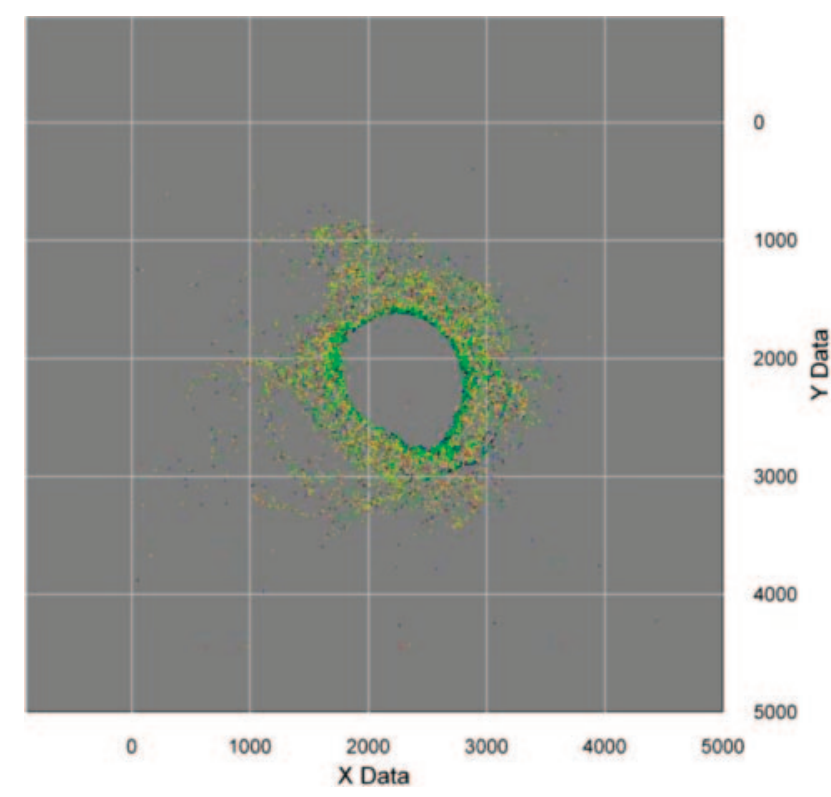

Fig. 7 Plot for circular feature showing debris size against position for a sample machined under DI water immersion range from the feature edge of between 75 and $150 \mu \mathrm{m}$. Outside this boundary the concentrations of debris drops significantly. This represents a significant reduction in the range of the debris when machined under liquid immersion, supporting the theory proposed by Fabbro et al. [32] that liquid immersion constricts the growth of the ablation plume. Even so, the use of DI water in this experiment has not prevented deposition of the debris back onto the substrate. According to Bowling [12], this could be a result of the lack of electrostatic conduction offered by the liquid medium chosen for this experiment, allowing the debris to be attracted back to the substrate surface by long-range electrostatic forces. The trails of debris that do lie outside the perimeter boundary evident in Fig. 5(b) follow what appear to be liquid flow lines and only lie downstream of the sample; thus it would appear that a low flow rate, provided by just $1 \mathrm{~m}$ of standing head pressure has affected the deposition pattern of the debris. The occurrence of these ripple patterns of debris deposition support the result of Sattari et al. [33], that proposed an increased flow-rate-promoted production rate of laser-ablated nanoparticles, claiming increased flow rates remove particles from the path of oncoming pulses more efficiently. Because of this, it is proposed that the use of higher flow rates will be interesting in combating debris deposition.

\subsection{Square features}

Figure 8 shows that when laser ablation machining in ambient air, the geometry of the feature being machined is critical to the positional deposition trend of the debris produced. To this end, machining square features is useful in illustrating the effect immersion machining has upon the homogeneity of the deposition distribution of debris. The difference between the data plot of the sample machined in ambient air (see Fig. 9) and the sample machined under liquid immersion (see Fig. 10) is even more pronounced than that of the circular features described above.

As Fig. 9 shows in detail, the sample generated in ambient air causes debris to be deposited with a

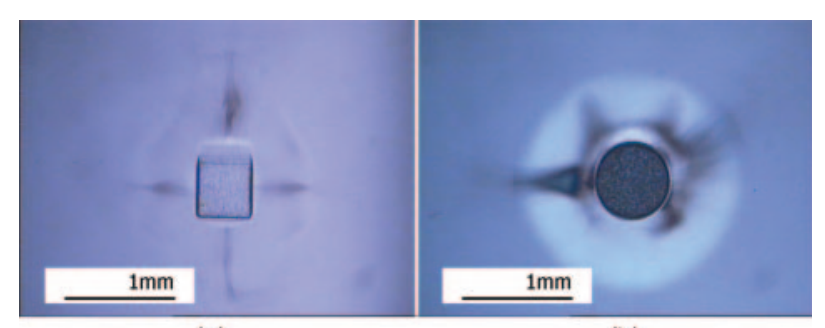

(a)

(b)

Fig. 8 A square and round feature machined in ambient air 


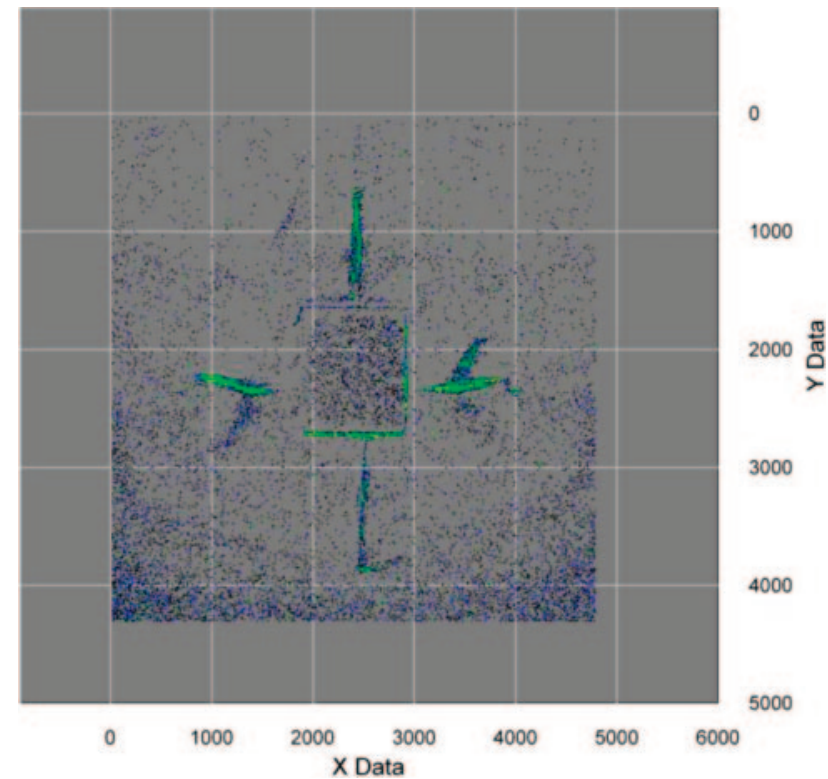

Fig. 9 Plot for square feature showing debris size against position for a sample machined in ambient air

modal tendency at a normal to the straight edges of the square feature. Thereafter, the debris mushroom at a distance of approximately $460 \mu \mathrm{m}$ from the feature centre. This modal response is highly pronounced for larger debris, the signal response of debris lying in the deposited streaks show a majority population of items between 2 and 17 square pixels. As with the circular features discussed above, this finding supports the proposal that debris transport is governed by the shockwave that has been reported to exist at the edge of the ablation plume $[\mathbf{1 3}, 15-17$, 37]. Debris is deposited at the extent of the ablation shockwave; as debris is liberated from the substrate during ablation by photomechanical processes, the solid and liquid species become trapped in a shockwave that is motivated by the gaseous products of photochemical interactions between the beam and material [25]. The patterns generated suggest the range is either governed by the point at which one shockwave meets another, each shockwave emanating from a neighbouring corner of the feature as described schematically in Fig. 11, or by the pulse length and fluence of the ablating beam $[39,40]$. The regime of the ablation plumes that create the debris patterns with a mushroom-like geometry, as one can see in Figs 8(a) and 9, and compared to the different deposition pattern of Fig. 8(b), are represented schematically in Fig. 11.

By comparison, the sample machined under liquid immersion, shown in Fig. 10, displays none of the modal trends of the dry sample, shown in Fig. 9. The range of debris deposition is limited to approximately half that of the sample machined in ambient air for

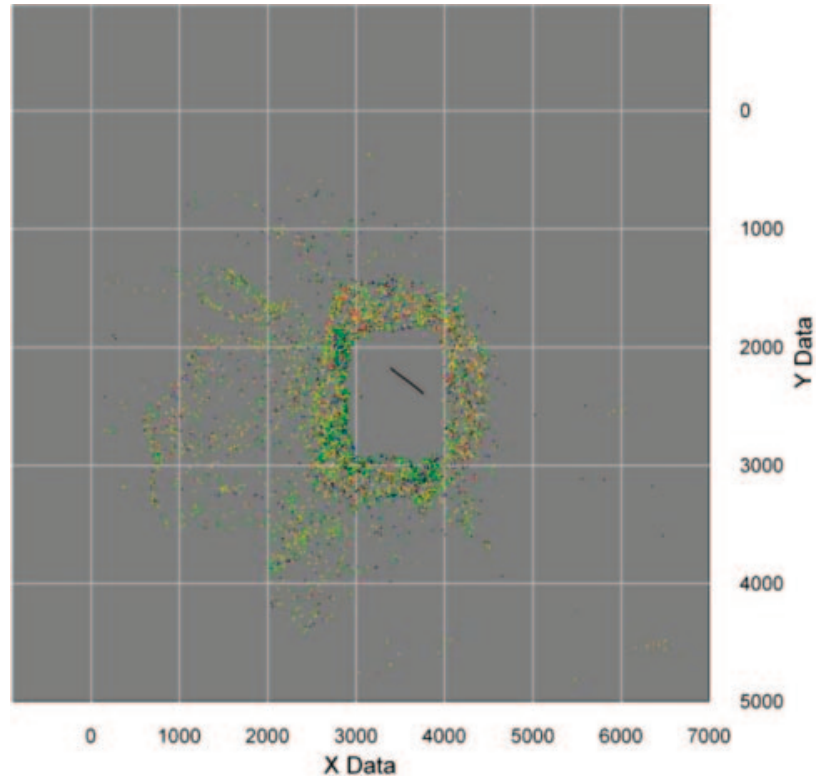

Fig. 10 Plot for square feature showing debris size against position for a sample machined under DI water immersion

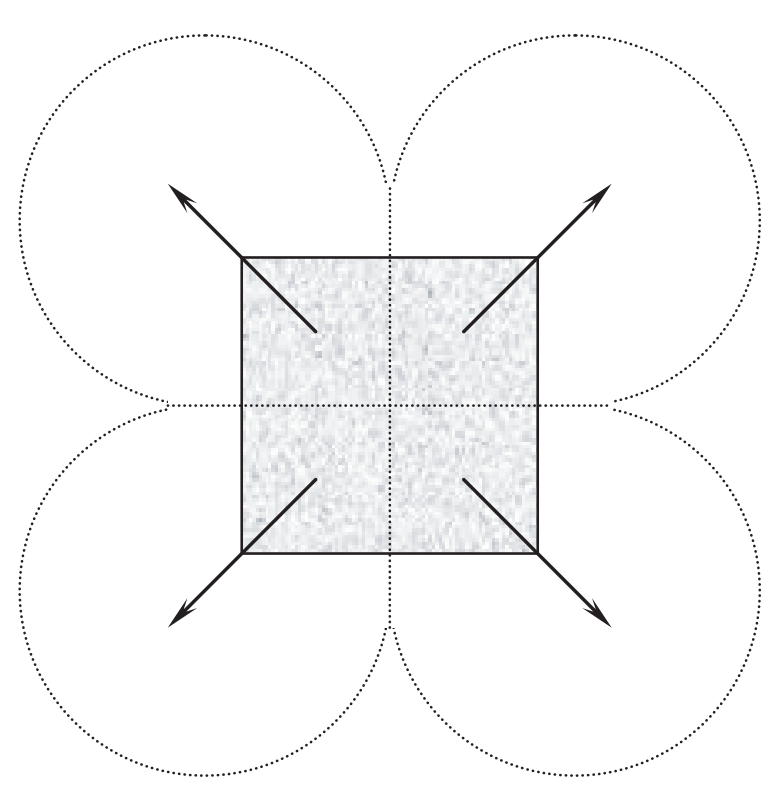

Fig. 11 A schematic illustrating four ablation plumes emanating from the corners of the square image. Debris lands in higher concentrations where they meet

the vast majority of species deposited and the particles appear to be homogeneously and randomly scattered within that range, again supporting the proposal of strong restriction of ablation plume expansion by the immersing liquid. Both the lack of deposition pattern and limited range are in agreement with the findings of Fabbro et al. [32]. Here the 
action of constricting the ablation plume will lead to higher temperatures, pressures, and turbulence within the plume. This in turn will lead to a more random and homogeneous deposition pattern shown in Fig. 10. The only modal trends that appear in Fig. 10 resemble fluid flow lines, supporting the findings of the circular sample detailed above and suggesting that the laminar flow of liquid has had an effect on the likely deposition site of generated debris. The common occurrence of debris downstream of the machined feature and very little debris upstream, observed in Fig. 10, provide further conformation and agreement with the work of Sattari et al. [33]

\subsection{Mean deposition size and size trends}

Comparison between the control sample of bisphenol A polycarbonate machined in calm, ambient air and the equivalent sample machined under immersion showed marked differences. The most apparent of these was the difference in size of the debris produced by each machining method. Inspection of Fig. 6 and Fig. 9, together representing machining conducted in ambient air in contrast to Fig. 7 and Fig. 10, together representing machining under thin laminar flow liquid immersion, it is clear that the vast majority of debris generated by ablation in ambient air have areas covering less than 17 square pixels. In contrast, the vast majority of the debris produced under liquid immersion covers an area greater than 16 square pixels. This is immediately apparent by the difference in shade between the two groups of plots: the dry samples, displayed in Fig. 6 and Fig. 9, are dominated by darker data point colours and the immersion samples, displayed in Fig. 7 and Fig. 10, are dominated by brighter data points and a large number of 64-129 pixel area response result data points.

This result can be illustrated in more quantative terms by measuring the mean size of debris produced using both machining techniques. Debris generated during immersion ablation increases in cross-sectional area by an order of magnitude from debris generated in ambient air, which was measured to be 4 and 3 square pixels in area for circular and square features respectively, to 33 and 71 square pixels for circular and square features respectively, generated under laminar flow DI water immersion.

Figures 12 and 13 plot the frequency of occurrence of debris features within ten size classes defined by $f=2^{n}-\left(2^{n}-1\right)$ for circular and square features respectively. The smallest debris detected is approximately five times more common on ambient air samples. Equally important is the increase in generation of large debris by immersion ablation. It is proposed that this is due to colloidal debris

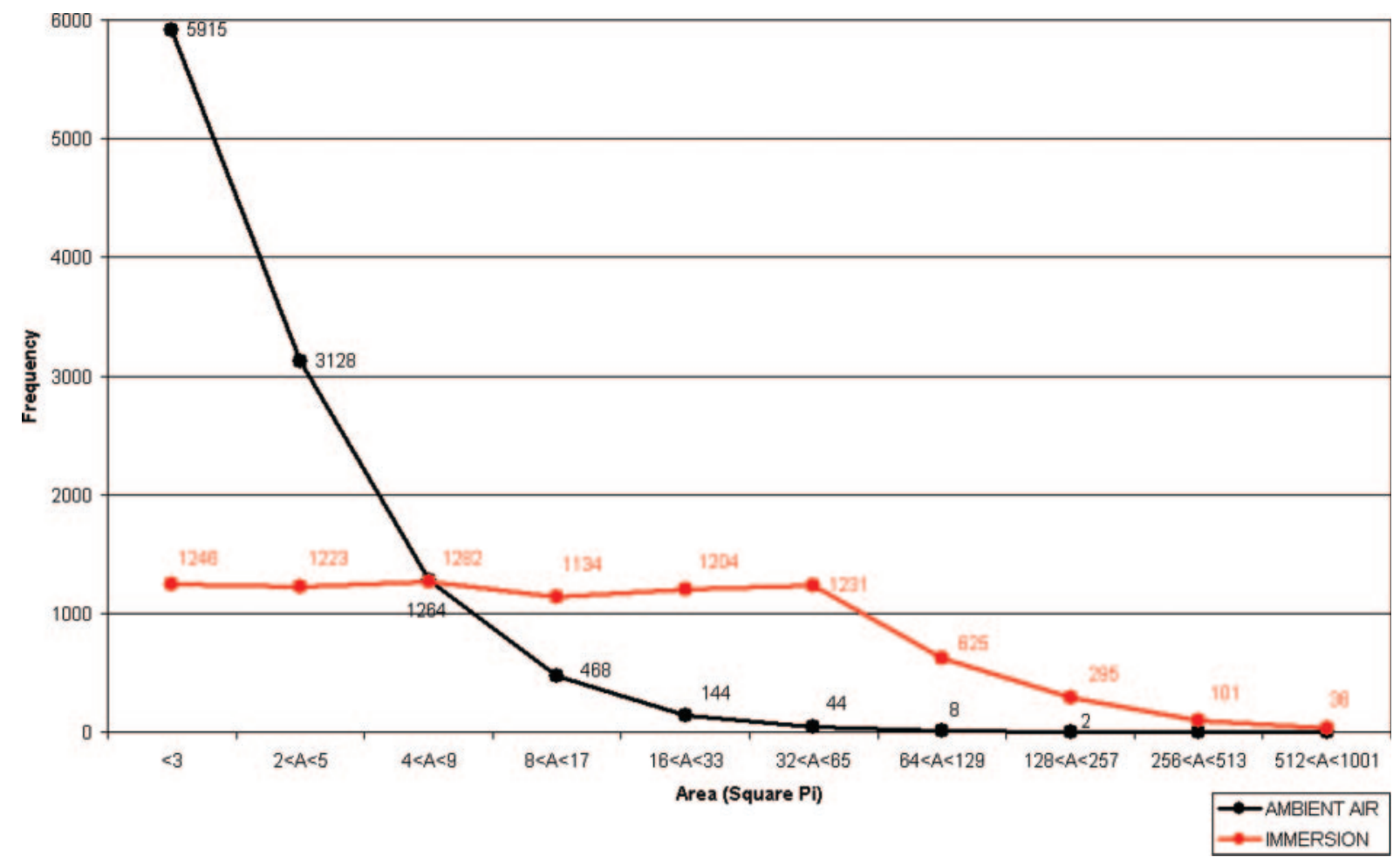

Fig. 12 Plot of frequency of debris lying within size classed groups for circular features 


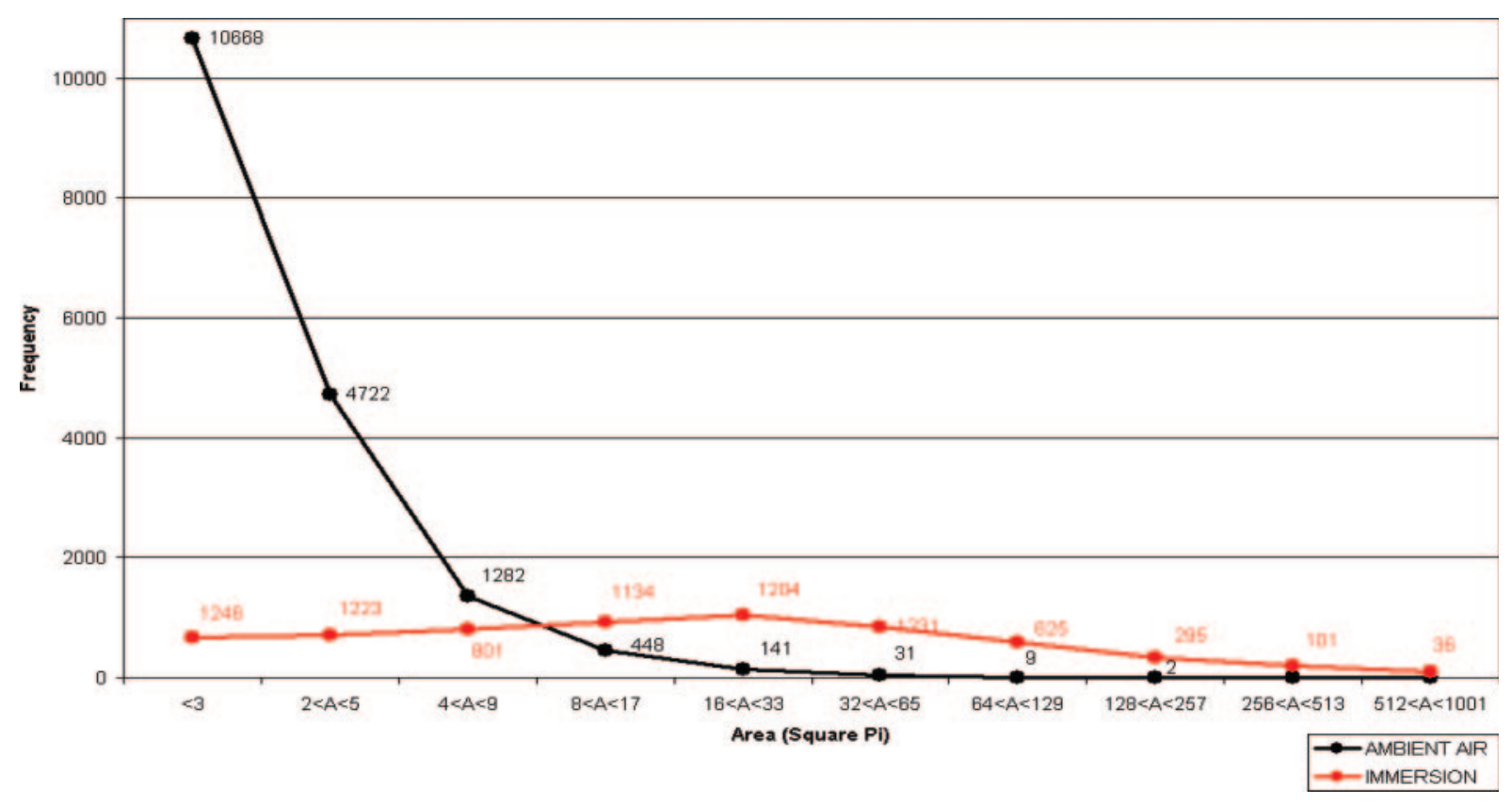

Fig. 13 Plot of frequency of debris lying within size classed groups for square features

agglomeration, as described by Heim et al. [41]. This theory offers satisfactory explanation for both the lack of small debris and the increased numbers of larger debris classes since the effects of colloidal adhesion, where microbubbles in the immersion medium collect populations of small debris particles at the gas liquid interface of the bubble, which are then forced together into a single, large particle as the microbubble collapses. This analysis supports the mean average values, with trends distributed about the mean values listed above. The numbers of small debris existent on the samples machined under liquid immersion are small compared to the samples machined in ambient air, but conversely, the numbers of intermediate and large debris existent on the samples machined under liquid immersion are high when compared to the samples machined in ambient air. This is the expected response for colloidal agglomeration, where large numbers of small debris are used to form smaller numbers of large particles. The production of larger debris with the use of immersion via action of colloidal interaction between debris items is in agreement with the results of Kato et al. [36], who noted the production of spongelike artefacts produced at high pulse fluences for stagnant-fluid-immersed laser-ablated targets.

\subsection{Feature geometry considerations}

Figures 5(a) and (b) show a stark contrast between the two features machined in terms of geometric accuracy. As detailed by Dowding and Lawrence [35], the ablation rate and ablation threshold of features when using liquid immersion in a thin film regime, as detailed by Zhu et al. [34], shows a significant loss in machining efficiency compared to ablation machining in ambient air. Also, the use of immersion ablation has been shown to considerably affect the topography of the features machined in terms of surface roughness [35]. This is thought to be due to a shift in primary etching mechanism. The immersing fluid has a higher density and viscosity than that of a typical gaseous machining medium, thus constricting the ablation plume expansion and therefore providing an inversely proportional increase in plume pressure, density, and temperature. This increase in plume density offers greater Bremsstrahlung attenuation to following laser pulses, reducing the laser etch rate. Simultaneously, the increased temperature and pressure of the ablation plume acts to etch the surface of the sample in a way not possible when machining in ambient air. However, when using thin film immersion the volume of the liquid covering the ablation plume is not sufficient to constrain the ablation plume at high pressure and the plume pressure is released in the action of splashing of the immersing fluid; thus, the etch rate achieved by the ablation plume is significantly reduced when compared to a thick film regime. The action of the plume etching, coupled with the random nature of immersion fluid splashing given the rippled nature of the flow over the sample gives poor geometric accuracy. The use of a new immersion rig to eradicate the optical effect of flow ripple over the top of the sample during machining will also prevent splashing of the immersing fluid and allow an increase of flow 
rate which is desired for better control of debris as stated above.

\section{CONCLUSIONS}

The feasibility, effects, and potential of debris control using laminar flow liquid immersion when machining with a KrF excimer laser have been examined. The ability to machine features of circular and square geometry through thin laminar flows has been demonstrated. Increased geometric accuracy of the features machined can be achieved by addressing the defocusing effect of flow ripples.

The positional debris deposition of samples machined in ambient air showed a modal tendency reliant on the feature shape machined. It is likely this is the result of the interaction of multiple shockwaves at the extent of ablation plumes generated at geometryspecific locations in the feature. Circular features typically leave a horn-shaped smear extending from the perimeter of the machined sample. Square features leave large mushroom-shaped smears lying at a normal to the edges of the feature machined. The results reveal that a four-leaf-clover-shaped ablation plume is the cause of the square feature smearing pattern since this plume shape yields a separate shockwave emanating from each corner of the feature; debris is deposited where the shockwaves collide, to consequently produce the mushroom smear pattern.

Laser ablation machining in ambient air has been shown to deposit debris with respect to species size. Large debris, measured to be between 16 and 65 square pixels, has small range, not travelling much further than the boundary of the machined feature; intermediate debris, classified as items returning a signal of between 4 and 17 square pixels, travels significantly further, being found in radial streaks at a normal to the circular features perimeter. This finding suggests shockwave-driven transport.

Laminar flows of liquid have shown potential to modify the end position of the debris produced: the use of immersion has reduced typical range by 17 per cent from $180 \mu \mathrm{m}$ from the feature boundary in ambient air to a concentrated area between 75 and $150 \mu \mathrm{m}$ from the feature edge under liquid immersion. The homogeneity of debris density was found to be increased by use of immersion ablation, with deposition within the boundary of the ablation plume having a comparatively even population density with respect to the sample machined in ambient air. This was particularly evident when comparing the square features machined in ambient air and under laminar flow DI water immersion. The ability to restrict the range of debris offers possibilities for dynamic optic protection to those implementing laser micromachining where optics must be positioned close to the sample.

Outside the evident ablation plume extents the evidence of positional control of deposited debris species by laminar flow DI water immersion were more pronounced. Both circular and square features displayed debris deposited in rippled flow line patterns, indicating the action of transport by fluid flow. This was achieved by the use of very low flow rates caused by a standing head pressure of just $1 \mathrm{~m}$. This result demonstrates that increased flow rates coupled with the eradication of defocusing surface ripples should offer better geometric feature accuracy with greater debris control. Furthermore, liquids with greater conducting properties would aid in preventing deposition on the machined workpiece.

The ability of laminar flow DI water immersion ablation to restrict the expansion of the ablation plume was demonstrated by the 17 per cent reduction in range of the debris produced and the clear outline of fine debris boundaries left on the samples produced by immersion ablation when compared to traditional ablation in gaseous mediums.

Laminar flow DI water immersion ablation has shown an ability to significantly increase the size of the debris species produced by laser ablation machining. Inspection of the mean debris size produced in ambient air revealed a value of 4 and 3 square pixels for circular and square features respectively. The same two features machined under DI water returned signals of 33 and 71 square pixels. This result was confirmed by inspection of the population spread of debris according to size. The samples machined in ambient air produced vast numbers of small debris and very few intermediate or large species, whereas samples machined under liquid immersion produced a quarter of the number of small debris, but the same amount of intermediate debris with very few large debris. This result was inline with a colloidal interaction response. The ability to produce larger, colloidal-type debris that has a homogenous deposition trend, rather than small particles that tend to be deposited in small areas may present benefits to sputter coating techniques. Also, the production of large debris items poses the potential for more efficient post-process cleaning: more removal force can be applied to larger debris items than to small species.

\section{REFERENCES}

1 Yung, K. C., Mei, S. M., and Yue, T. M. Pulsed UV laser ablation of a liquid crystal polymer. Int. J. Adv. Mfg Technol., 2005, 26(11), 1231-1236.

$2 \mathbf{X u}, \mathbf{X} . \mathbf{F}$. Phase explosion and its time lag in nanosecond laser ablation. Appl. Surf. Sci., 2002, 197, 61-66. 
3 Xu, X. F. and Song, K. H. Phase change phenomena during high power laser-materials interaction. Mater. Sci. Engng A-Struct. Mater. Props Microstruct. Process., 2000, 292(2), 162-168.

$4 \mathbf{X u}, \mathbf{X}$. F. and Song, K. H. Interface kinetics during pulsed laser ablation. Appl. Phys. A-Mater. Sci. Process., 1999, 69(Suplement 1), S869-S873.

5 Prasad, M., Conforti, P. F., and Garrison, B. J. Coupled molecular dynamics-Monte Carlo model to study the role of chemical processes during laser ablation of polymeric materials. J. Chem. Phys., 2007, 127(8), 337-349.

6 Cooper, J. B., Julian, B., Morrison, H., Song, P., Albin, S., and Zehn, J. L. Surface characterization of pulsed UV-laser modified polyamide films. Thin Solid Films, 1997, 303(1-2), 180-190.

7 Révész, K., Ho, B., and Borr, Z. Excimer laser induced surface chemical modification of polytetrafluoroethylene. Appl. Surf. Sci., 1996, 109, 222-226.

8 Lee, S. K., Chang, W. S., and Na, S. J. Numerical and experimental study on the thermal damage of thin Cr films induced by excimer laser irradiation. J. Appl. Phys., 1999, 86(8), 4282-4289.

9 Pataulf, G. and Dyer, P. Photomechanical processes and effects in ablation. Cheml. Rev., 2003, 103(2), 487-518.

10 Georgiou, S. and Koubenakis, A. Laser-induced material ejection from model molecular solids and liquids: mechanisms, implications and applications. Cheml. Rev., 2003, 103(2), 349-394.

11 Kelly, R. and Miotello, A. On the role of thermal processes in sputtering and composition changes due to ions or laser pulses. Nucl. Instrum. Methods Phys. Res. S B: Beam Interact. Mater. Ats., 1998, 141(1-4), 49-60.

12 Bowling, R. A. A theoretical review of particle adhesion. In Particles on surfaces: detection, adhesion, and removal, (Ed. I. K. L. Mittal), 1988, pp. 129-142 (CRC Press, New York).

13 Dyer, P. E., Farrar, S., and Key, P. H. Investigation of excimer ablation of ceramic and thin film Y-Ba-Cu-O using nanosecond photoacoustic techniques. Appl. Phys. Lett., 1992, 60(15), 1890-1892.

14 Lippert, T., David, C., Dickinson, J. T., Hauer, M., Kogelschatz, U., Langford, S. C., Nuyken, O., Phis, C., Robert, J., and Wokaun, A. Structure-property relations of photoreactive polymers designed for laser ablation. J. Photochem. Photobiol. A, 2001, 145(3), 145-157.

15 Dyer, P. E., Karnakis, D. M., Key, P. H., and Tait, J. P. Excimer laser ablation of low and high absorption index polymers. Appl. Surf. Sci., 1996, 96-98(2), 167-173.

16 Dyer, P. E., Karnakis, D. M., Key, P. H., and Sands, D. Fast photography of UV laser ablated metal films. Appl. Surf. Sci., 1997, 109-110, 168-173.

17 Clarke, P., Dyer, P. E., Key, P. H., and Snelling, H. V. Plasma ignition thresholds in UV laser ablation plumes. Appl. Phys. A, 1999, 69(7), S117-S120.

18 Kruusing, A. Underwater and water-assisted laser processing: part 2 - etching, cutting and rarely used methods. Opt. Lasers Engng., 2004, 41(2), 329-352.

19 Yukawa, H. Quantum theory of non-local fields. Part 1. Free fields. Physi. Rev., 1950, 77, 219-226.

20 Kim, J. Y., Sohn, D., and Kim, E. R. Polymer-based multi-layer conductive electrode film for plastic LCD applications. Appl. Phys. A, 2001, 72(6), 699-704.
21 Bardina, J. Methods for surface particle removal: a comparative study. Particulate Sci. Technol., 1988, 6(2), 121-131.

22 Zapka, W., Asch, K., Keyser, J., and Meissner, K. Removal of particles from solid-state surfaces. European patent EP 0297506 A2, 04/01/1989.

23 Rizvi, N. H., Rhumsby, P. T., and Gower, M. C. New developments and applications in the production of 3D microstructures by laser micromachining. Proc. SPIE, 3898, 1999, 240, pp. 240-249.

24 Ashfold, M. N. R., Claeyssens, F., Fuge, J. M., and Henley, S. J. Pulsed laser ablation and deposition of thin films. Chem. Soci. Rev., 2004, 33, 23-31.

25 Krupp, H. Particle adhesion theory and experiment. Adv. Colloid Interface Sci., 1967, 1(2), 111-239.

26 Visser, J. Measurement of the force of adhesion between submicron carbon-black particles and a cellulose film in aqueous solution. J. Colloid Interface. Sci., 1970, 34(1), 6-31.

27 Bhattacharya, S. and Mittal, K. L. Mechanics of removing glass particles from a solid surface. Surf. Technol., 1978, 7, 413-425.

28 Biggs, S., Cain, R. G., Dagastine, R. R., and Page, N. W. Direct measurements of the adhesion between a glass particle and a glass surface in a humid atmosphere. J. Adhes. Sci. Technol., 2002, 16, 869-885.

29 Leite, F. L. and Herrmann, P. S. P. Mapping of adhesion forces on soil minerals in air and water by atomic force spectroscopy (AFS). J. Adhes. Sci. Technol., 2003, 17(16), 2141-2156.

30 Vezenov, D. V., Noy, A., and Ashby, P. Chemical force microscopy. Ann. Rev. Mater. Sci., 1997, 27(1), 381-421.

31 Yang, G. W. Laser ablation in liquids: applications in the synthesis of nanocrystals. Prog. Mater. Sci., 2006, 52(4), 648-698.

32 Fabbro, R., Peyre, P., Berthe, L., and Scherpereel, X. L. Physics and applications of laser-shock processing. J. Laser Appl., 1998, 10(6), 265-279.

33 Sattari, R., Sajti, C. L., Kahn, S., and Barcikowski, S. Scale-up of nanoparticle production during laser ablation of ceramics in liquid media. In Proceedings of the 27th International Congress on Application of lasers and electro-optics: laser materials processing section, Temecula, California, 2008, pp. 49-54 (Laser Institute of America, Orlando, Florida).

34 Zhu, S., Lu, Y. F., Hong, M. H., and Chen, X. Y. Laser ablation of solid substrates in water and ambient air. J. Appl. Phys., 2001, 89(4), 2400-2403.

35 Dowding, C. F. and Lawrence, J. Analysis of the excimer laser ablation characteristics of bisphenol A polycarbonate in ambient air and under thin film laminar flow water immersion. In Proceedings of the 27th International Congress on Application of lasers and electro-optics: laser materials processing section, Temecula, California, 2008, pp. 202-211 (Laser Institute of America, Orlando, Florida).

36 Katto, M., Kuroe, Y., Kaku, M., Kubodera, S., Yokotani, A., Katayama, H., and Nakayama, T. Nano-particles of hydroxyapatite formed by underwater laser ablation method. In Proceedings of The 27th International Congress on Application of lasers and electro-optics: laser materials processing section, 
Temecula, California, 2008, pp. 65-67 (Laser Institute of America, Orlando, Florida).

37 Dyer, P. E., Key, P. H., Sands, D., Snelling, H. V., and Wagner, F. X. Blast-wave studies of excimer laser ablation of ZnS. Appl. Surf. Sci., 1995, 86(1), 18-23.

38 Kawamoto, H. The history of the liquid-crystal displays. Proc. IEEE, 2002, 90(4), 460-500.

39 Berthe, L., Fabbro, R., Peyre, P., and Bartnicki, E. Wavelength dependence of laser shock-wave generation in the water-confinement regime. J. Appl. Phys. 1999, 85(11), 7552-7555.
40 Peyer, P., Berthe, L., Fabbro, R., and Sollier, A. Experimental determination by PVDF and EMV techniques of shock amplitudes induced by 0.6-3 ns laser pulses in a confined regime with water. J. Phys. D. Appl. Phys., 2000, 33(5), 498-503.

41 Heim, L. O., Ecke, S., Preuss, M., and Butt, H. J. Adhesion forces between individual gold and polystyrene particles. J. Adhes. Sci. Technol., 2002, 16(7), 829-843. 